\title{
Cálculo del balance energético de una plantación de Populus deltoides clon Lux con fines energéticos en un sitio con ambiente mediterráneo
}

\author{
Energy balance in a bioenergy plantation of Populus deltoides clone Lux in a site with \\ Mediterranean environment
}

\author{
Antonio Cabrera ${ }^{\text {a*}}$, Cristiano Tozzini ${ }^{\mathrm{b}}$, Sergio Espinoza ${ }^{\mathrm{a}}$, Rómulo Santelices ${ }^{\text {a }}$, Enrico Bonari ${ }^{\mathrm{b}}$ \\ *Autor de correspondencia: ${ }^{a}$ Universidad Católica del Maule, Centro de Desarrollo del Secano Interior, \\ Avenida San Miguel 3605, Talca, Chile, tel.: 071-2413728, acabrera@ucm.cl \\ b Scuola Superiore Santa Anna, Pisa, Italia.
}

\begin{abstract}
SUMMARY
Given the energy challenges confronted by the world in general, where Chile is not an exception, forest energy crops have been positioned as a viable alternative to diversify this country's energy matrix. These types of crops, still under development, are based on short rotation shifts with high biomass production. The problem arises when assessing the sustainability of such crops. Accordingly, the energy balance is an important factor to assess sustainability i.e. we cannot spend more energy to produce biomass than what is released by the biomass combustion. This work presents the energy balance of harvest products at roadside in a Populus deltoides clone Lux short rotation forestry during eight years (2000-2008), comparing biennial (T2) and triennial (T3) shifts. Consequently, we calculated the energy cost of production and harvest and the amount of energy released in the combustion of the biomass generated. Both rotation shifts got positive results, being triennial shifts more energy efficient than biennial ones (34.7 T2 vs 25 T3).
\end{abstract}

Key words: biomass, sustainability, short rotation forestry.

\section{RESUMEN}

Ante el desafío energético al cual se enfrenta el mundo en general, y Chile no es una excepción, los cultivos forestales con destinación energética se han ido posicionando como una alternativa viable para la diversificación de la matriz energética del país. Este tipo de cultivos, aún en fase de desarrollo, se basan en turnos cortos de rotación con altas producciones de biomasa. El problema surge a la hora de evaluar la sostenibilidad de dichos cultivos. El cálculo del balance energético es clave para valorar dicha sostenibilidad, es decir, no se puede gastar más energía en producir biomasa que la que esta otorga con la combustión. En este trabajo se calcula el balance energético, considerando los productos de la cosecha puestos a orilla de camino, de un cultivo de Populus deltoides clon Lux con fines energéticos durante un periodo de ocho años (2000-2008), comparando un turno de rotación bienal (T2) con uno trienal (T3) en un sitio con ambiente mediterráneo. Para ello se ha calculado el costo energético de la producción y cosecha, y la cantidad de energía liberada en la combustión de la biomasa generada. En ambos turnos de rotación se obtienen resultados positivos, siendo más conveniente energéticamente el turno trienal respecto al bienal (34,7 T2 vs 25 T3).

Palabras clave: biomasa, sostenibilidad, cultivos de corta rotación.

\section{INTRODUCCIÓN}

En el transcurso de los últimos años el fuerte incremento demográfico registrado a nivel global se ha traducido en una creciente necesidad de energía, tanto en países desarrollados como en aquellos en vía de desarrollo. Una de las fuentes más prometedoras para la producción de energía es la biomasa, más concretamente los cultivos lignocelulósicos que pueden ser utilizados para producir calor y electricidad a través de la combustión directa o la producción de biofuel o biogás a través de la pirólisis y gasificación (Mantineo et al. 2009, Sevigne et al. 2011). La energía proveniente de la biomasa, tanto lignocelulósica como herbácea, se presenta como una alternativa energética sostenible y amigable con el medio ambiente; de hecho, se considera que la energía obtenida a partir de biomasa no tiene emisiones de carbono, ya que el carbono emitido en la combustión es el mismo que las plantas absorben mientras crecen (The Royal Society 2008).

Un importante problema relacionado con el uso de cultivos alternativos para la producción de energía es la evaluación de la sostenibilidad ambiental de dichos cultivos a largo plazo (Bonari et al. 1992). A tal propósito, los balances agroambientales, y más concretamente el balance energético, representan un instrumento adecuado para la evaluación de dicha sostenibilidad. Idealmente los cultivos 
energéticos deberían dar altas producciones de biomasa y por tanto de energía, siendo menor el gasto energético empleado para su producción que el que se contiene en dicha biomasa. A diferencia de los cultivos anuales, los perennes tienen la ventaja de poder distribuir los costos de plantación (se planta solo el primer año y luego se manejan los rebrotes), entendiendo estos costos como energéticos, no como económicos, durante el periodo de cultivo, obteniendo así una relación output/input (egreso/ingreso) más favorable (Angelini et al. 2005).

El balance energético pone de manifiesto la energía producida por unidad de energía empleada en el proceso de producción y transformación de la biomasa en energía eléctrica (Scholz 2002). Dicho balance ha sido ampliamente usado en diferentes trabajos (Bonari 1992, Dubuisson y Sintzoff 1998, West y Marland 2002, Heller 2003, Sartori 2005, Mead 2006, Boehmel 2008, Gasol 2009, Nassi o Di Nasso et al. 2010). Dubuisson et al. (1998), por ejemplo, calcularon el balance energético para un cultivo de Рориlus sp. con destinación energética en Bélgica, analizando tres niveles de intensificación cultural e incluyendo el transporte de la biomasa a la central de transformación. Los resultados muestran una relación output/input de 22, 23 y 26 para los tres niveles de intensificación. Heller et al. (2003), sin embargo, calcularon el balance energético para un cultivo de Salix sp. con fines energéticos en Nueva York, alcanzando una relación output/input que varió entre 58 y 40 en función de las dosis de fertilizante aplicadas. Otros estudios (e.g. Mead et al. 2006), han obtenido un balance energético para plantaciones de Eucalyptus grandis Hill ex maiden y Pinus taeda Linn, calculando una relación output/input variable en función de los medios técnicos utilizados y con un valor cercano a 25 .

En el caso del cultivo de especies del género Populus, la mayor parte de los datos sobre los balances energéticos se basan en previsiones y no en condiciones ambientales reales (Bonari y Villani 2004). Para mejorar la eficiencia energética es necesario entender la influencia en la gestión silvícola del cultivo, comparando diferentes turnos de corta o rotación, el empleo de diversas dosis de fertilizante, diferentes densidades de plantación y diferentes alternativas de preparación del terreno (Angelini et al. 2005, Sartori 2005, Boehmel 2008). En el caso de Chile, país donde el cultivo de híbridos del género Populus podría tener un enorme potencial de desarrollo, aún no se han hecho estudios sobre el balance energético en este tipo de cultivos energéticos del género Populus; de hecho, Baettig et al. (2010) sugieren que habría que considerar el consumo energético ligado a la fabricación de pesticidas, herbicidas, fertilizantes, lubricantes, insumos variados, emisión directa por quema de combustibles durante el laboreo, consumo energético del bombeo de agua de riego, entre muchas otras. Algunos de estos datos se consideraron a la hora de realizar el cálculo del balance energético de este estudio. Hay diversas formas de aproximación al estudio del balance energético y todavía no hay un consenso a nivel inter- nacional de cuáles inputs deben ser incluidos en el cálculo. De hecho, se ha podido observar una gran variación en los coeficientes energéticos y los límites del sistema a analizar. Como ejemplo, Dubuisson y Sintzoof (1998) estudiaron la producción de Populus spp. con tres niveles de intensificación en el cultivo (bajo, medio y alto input) hasta la producción de astillado. Obtuvieron valores que variaron de 6,9 a 7,5 GJ ha ${ }^{-1}$ año-1 para los diferentes inputs, 168 a 226 $\mathrm{GJ} \mathrm{ha}^{-1}$ año ${ }^{-1}$ para los output y de 23 a 30 para la eficiencia energética. Scholz (1998) presenta un balance energético para el cultivo de Populus spp. con fines energéticos cuya configuración va desde la preparación del terreno hasta el transporte de la biomasa a la central de transformación, en este caso con unos inputs reducidos debido principalmente a la ausencia de fertilización. Sin embargo, la relación output/input ha resultado bastante baja debido a la inclusión del transporte y almacenamiento. En el caso de Dillen et al. (2013), el resultado del balance también fue positivo, obteniendo 7,9 veces más energía que la consumida, incluyendo todas las operaciones de cultivo de álamo y realizando el análisis del balance a orilla de camino.

Este trabajo plantea la hipótesis de que el balance energético en el cultivo de Populus deltoides Marshall clon Lux con destinación energética con turnos de rotación bianual y trianual en un ciclo de ocho años, es positivo, es decir, la energía que se obtiene es mayor que la energía empleada en todo el proceso productivo. El objetivo del estudio es estimar el balance energético y la producción de biomasa de un cultivo de $P$. deltoides clon Lux durante un ciclo de cultivo de ocho años, comparando turnos de corta bienal y trienal.

\section{MÉTODOS}

Área de estudio. El estudio se ubicó en el Centro di Ricerche Agro-ambientale (CIRRA) Enrico Avanzi de la Universidad de Pisa (Italia). Se analizó un cultivo de $P$. deltoides clon Lux, establecido en un sitio, gestionado con dos diferentes turnos de rotación o corta, bienal y trienal. Los campos experimentales están situados en San Piero A Grado, a $43^{\circ} 40^{\prime} \mathrm{N}, 10^{\circ} 21^{\prime}$ E, a $5 \mathrm{~m}$ s.n.m. y a $2 \mathrm{~km}$ en línea de aire de la costa. Los datos meteorológicos para la caracterización climática del sitio se tomaron de la estación de San Piero A Grado que pertenece a la red nacional del Ufficio Centrale di Ecologia Agraria (UCEA) del Ministerio de Políticas Agrarias y Forestales ${ }^{1}$.

El experimento, realizado en dos parcelas de $5.000 \mathrm{~m}^{2}$ cada una, empezó en el año 2000 con la plantación de material certificado de estacas de P. deltoides clon Lux a una densidad de 10.000 plantas ha $^{-1}$ (2 m x 0,5 m), en terrenos de granulometría media con buena fertilidad agronómica y una capa freática no muy profunda (Bonari y Villani 2004). En el año 2002 se efectuó la primera cosecha en ambas parcelas y fue en ese año cuando se eligieron dos

CMA - Unita' di Ricerca per la Climatologia e la Meteorologia Applicate all'Agricoltura. http://cma.entecra.it/homePage.htm 
turnos de corta diferentes (bienal vs trienal). Cada turno de corta estuvo representado en una parcela individual siendo cada parcela la base de las futuras comparaciones. El bienal (T2) se cosechó en el invierno del 2002, 2004, 2006 y 2008 y el trienal (T3) en el invierno del 2002, 2005 y 2008.

El suelo fue preparado con anticipación a la temporada de plantación (octubre 1999), por medio de arado profundo a $50 \mathrm{~cm}$, seguido de un subsolado con camellón y otro arado. A continuación se aplicó herbicida pre-plantación (Terbutilazina, Click ${ }^{\circledR}$ 50, $3 \mathrm{Lha}^{-1}$ ) y una fertilización, usando $600 \mathrm{~kg} \mathrm{ha}^{-1}$ de fertilizante 8-24-24. En la plantación se utilizó un prototipo de máquina plantadora diseñado por Spapperi ${ }^{\circledR}$. Para la cosecha se hizo con una cosechadora-astilladora-cargadora diseñada y creada también por Spapperi ${ }^{\circledR}$ y un remolque llevado por tractor para la recogida del astillado. Después de cada cosecha se realizó una fertilización de cobertura aplicando $100 \mathrm{~kg} \mathrm{ha}^{-1} \mathrm{de} \mathrm{ni-}$ trógeno suministrado en forma de urea.

Al final del ensayo el terreno fue recuperado, eliminando los tocones con una destoconadora y realizando un arado profundo y un subsolado. Se usaron diversos tipos de tractores dependiendo de la operación a realizar: 132 $\mathrm{kW}$ de potencia para el arado y cosecha, $73 \mathrm{~kW}$ para el subsolado y $48 \mathrm{~kW}$ para las otras operaciones.

Producción de biomasa. Las muestras para el análisis de la biomasa se colectaron desde un mes antes del inicio del crecimiento vegetativo hasta el mes de febrero sucesivo, con una periodicidad mensual. Febrero se considera periodo óptimo para la cosecha de cultivos de corta rotación del género Populus con destinación energética en las latitudes donde se realizó la prueba (Bonari y Villani 2004). Las muestras, cuatro por cada turno de rotación y fecha de muestreo, se tomaron dentro de filas de 10 metros, y consistieron en plantas completas, eligiendo las que crecieron en competencia perfecta al interior de la plantación, descartando filas externas o próximas a zonas de mucha mortalidad. Para el cálculo de la biomasa, las diferentes muestras fueron introducidas en estufa a $60{ }^{\circ} \mathrm{C}$ hasta que alcanzaron peso constante, es decir, perdieron toda la humedad. La biomasa por hectárea para cada turno de corta se obtuvo de la media de los individuos seleccionados para cada turno de corta y multiplicado por la densidad de plantación.

Balance energético. Para la determinación del balance energético este estudio consideró los flujos de energía asociados a las operaciones necesarias para el cultivo de $P$. deltoides clon Lux, excluyendo la energía necesaria para el transporte del producto a la central de conversión, es decir, se ha realizado un balance energético a pie de campo u orilla del camino. La energía de mano de obra y producción de las estacas para la plantación no se tuvo en cuenta por ser inferior al 0,2 \% del total de la energía (Boehmel 2008).

En la determinación de los inputs energéticos necesarios para la realización del cultivo se consideraron:

- Los costos energéticos para la fabricación y reparación de las máquinas agrícolas.

- Los costos para la producción de los fertilizantes y herbicidas.

- Los costos por el consumo de combustible y aceite lubricante en las diferentes operaciones de cultivo.

Se asume que las máquinas y los aperos se usaron en 200 hectáreas y tienen una vida útil de 10 años (Bonari et al. 1999). Los costos energéticos para la construcción, depreciación y mantenimiento de los tractores se calcularon teniendo en cuenta la vida media y el tiempo de uso en cada operación de cultivo. Estos datos se transformaron en datos cuantitativos energéticos, utilizando coeficientes extraídos de la revisión bibliográfica internacional (cuadro 1). La producción de energía obtenida del sistema (output) fue determinada multiplicando la producción en materia seca por el poder calorífico de la biomasa de P. deltoides clon Lux (calculado usando la bomba calorimétrica de Mahler según el método ASTM D 2015). Sucesivamente, se calculó la producción de energía neta (output-input) y la eficiencia energética (output/input). Para el cálculo de la energía neta, se usó la siguiente fórmula [1]:

Output - Input $=$ Energía producida (poder calorífico $\mathrm{x}$ cosecha) - Energía consumida (operaciones + energía de los factores productivos)

Cuadro 1. Equivalentes energéticos de diferentes parámetros usados en el estudio. Energy conversion factors for several parameters used in the study.

\begin{tabular}{lccl}
\hline \multicolumn{1}{c}{ Parámetro } & Unidad de medida & Equivalentes energéticos & \multicolumn{1}{c}{ Referencias } \\
\hline Máquinas & $\mathrm{MJ} \mathrm{kg}^{-1}$ & 108 & Kalk (1996) \\
Combustible & $\mathrm{MJ} \mathrm{L}^{-1}$ & 42,7 & Boehmel (2008) \\
Aceite & $\mathrm{MJ} \mathrm{L}^{-1}$ & 80 & Bonari (1992) \\
Nitrógeno & $\mathrm{MJ} \mathrm{kg}^{-1}$ & 47,1 & Acaraglu(2005) \\
$\mathrm{P}_{2} \mathrm{O}_{5}$ & $\mathrm{MJ} \mathrm{kg}^{-1}$ & 15,8 & Kaltschmitt (1997) \\
$\mathrm{K}_{2} \mathrm{O}$ & $\mathrm{MJ} \mathrm{kg}^{-1}$ & 9,3 & Kaltschmitt(1997) \\
Herbicidas & $\mathrm{MJ} \mathrm{kg}^{-1}$ & 276 & West y Marland (2002 \\
\hline
\end{tabular}


Se creó una base de datos para determinar la energía empleada en la realización de cada una de las operaciones de cultivo, detallando el costo energético directo y el indirecto de las diferentes operaciones (siendo el costo directo el que se refiere al costo de la operación específica, mientras que el costo indirecto es el que se refiere al costo energético debido a la construcción del tractor y de los aperos). La mayor parte de los datos presentados en el cuadro 2 forman parte la base de datos del software Sisco, desarrollado para el cálculo de balances energéticos en el Centro Enrico Avanzi (Bonari 1999). Otros coeficientes se calcularon midiendo el consumo de los tractores en las diferentes operaciones de cultivo en pruebas de campo.

\section{RESULTADOS}

Producción de biomasa. Las medias anuales productivas en el estudio del ciclo completo del cultivo de P. deltoides clon Lux fueron de 9,2 Mgha $^{-1}$ año-1y 11,5 Mgha $^{-1}$ año $^{-1}$ para el turno bienal y trienal, respectivamente, para el período 2000 -2008 (figura 1).

Balance energético. El cálculo de los inputs (cuadro 3) en los dos turnos de rotación estudiados presentó un mismo valor en el primer año $\left(16,2 \mathrm{GJ} \mathrm{ha}^{-1}\right)$. Del total de los inputs, el $11 \%$ correspondió a costos indirectos relativos a la fabricación y mantenimiento de las máquinas agrícolas utilizadas, mientras que el $89 \%$ restante fueron costos energéticos directos relacionados con el uso de medios técnicos y al consumo de combustibles y lubricantes en las distintas operaciones de cultivo. En particular, el costo energético más elevado fue el del uso de fertilizantes que representan casi el 48\% del total de los inputs energéticos.

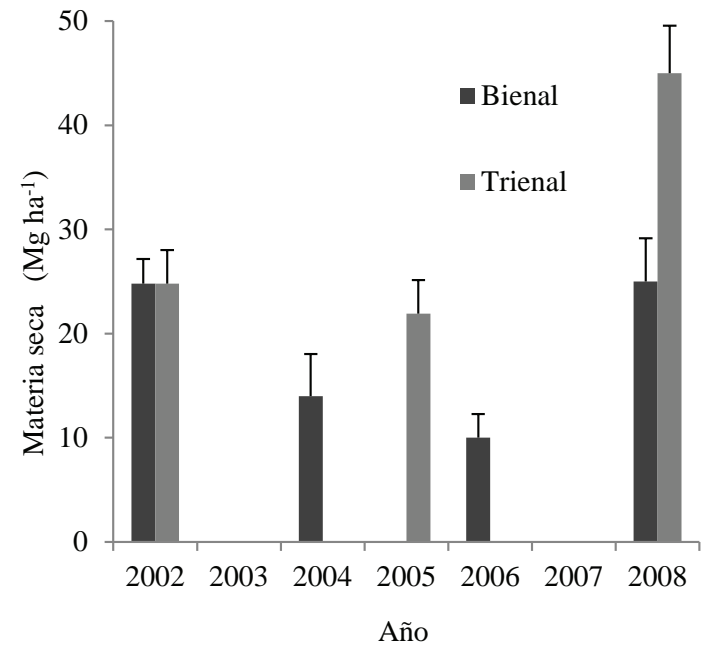

Figura 1. Producción de biomasa en el cultivo de Populus deltoides clon Lux en el periodo 2000-2008.

Populus deltoides clone Lux biomass production during the period 2000-2008.

Respecto al output, hay que señalar que el poder calorífico medio de la madera de Populus deltoides clon Lux para ambos turnos de rotación fue de 19,5 $\mathrm{MJ} \mathrm{kg}^{-1}$.

En función del turno de corta, los outputs obtenidos por el sistema variaron de un turno al otro, siendo el turno trienal el que más energía aportó (+21,5 \%). Los turnos de corta se caracterizaron por una diferente eficiencia energética, de hecho, con el turno bienal fue posible obtener $25 \mathrm{GJ} \mathrm{ha}^{-1}$ año ${ }^{-1}$ por cada gigajoule de energía introducido en el sistema, mientras que esta relación subió un 38,8 \% en el turno de corta trianual.

Cuadro 2. Energía directa e indirecta empleada en las operaciones de cultivo, fertilización, herbicidas y cosecha. Direct and indirect energy used in farming operations, fertilizer, herbicides and crop.

\begin{tabular}{|c|c|c|c|c|c|}
\hline \multirow{2}{*}{\multicolumn{2}{|c|}{ Operaciones }} & \multirow{2}{*}{ Tractor $(\mathrm{kW})$} & \multicolumn{3}{|c|}{ Energía consumida $\left(\mathrm{MJ} \mathrm{ha}^{-1}\right)$} \\
\hline & & & Directa & Indirecta & Total \\
\hline \multicolumn{2}{|c|}{ Arado profundo (40-50cm) } & 132 & $2.804,8$ & 479 & $3.283,8$ \\
\hline \multicolumn{2}{|l|}{ Subsolado } & 73 & 332 & 308 & 640 \\
\hline \multicolumn{2}{|l|}{ Arado } & 132 & 1.500 & 479 & 1.979 \\
\hline \multicolumn{2}{|l|}{ Aplicación fertilizante } & 48 & 334,6 & 161 & 495,6 \\
\hline \multicolumn{2}{|l|}{ Nitrógeno $\left(\mathrm{MJ} \mathrm{kg}^{-1}\right)$} & - & 47,1 & - & 47,1 \\
\hline \multicolumn{2}{|c|}{ Fertilizante 8-24-24 $\left(\mathrm{MJ} \mathrm{kg}^{-1}\right)$} & - & 12,7 & - & 12,7 \\
\hline \multicolumn{2}{|l|}{ Aplicación herbicida } & 48 & 303 & 164 & 467 \\
\hline \multicolumn{2}{|l|}{ Herbicida $\left(\mathrm{MJ} \mathrm{l}^{-1}\right)$} & - & 91,2 & - & 91,2 \\
\hline \multicolumn{2}{|l|}{ Plantación $P$. deltoides } & 48 & 800 & 210 & 1.010 \\
\hline Cosecha P. deltoides & $\mathrm{T} 2$ & 132 & 2.198 & 503 & 2.701 \\
\hline Cosecha $P$. deltoides & T3 & 132 & 3.449 & 503 & 3.952 \\
\hline \multicolumn{2}{|l|}{ Tractor + camión } & 123 & 709 & 719 & 1.428 \\
\hline \multicolumn{2}{|l|}{ Destoconadora } & 132 & 1.113 & 400 & 1.513 \\
\hline
\end{tabular}


Cuadro 3.Balance energético para el cultivo de Populus deltoides clon Lux con fines energéticos con turnos bienales (T2) y trienales (T3) para el período 2000-2008.

Biannual (T2) and triennial (T3) Populus deltoides clone Lux crop energy balance for the period 2000-2008.

\begin{tabular}{|c|c|c|c|c|c|c|c|c|}
\hline \multirow[b]{2}{*}{ Año } & \multicolumn{2}{|c|}{$\begin{array}{c}\text { Input } \\
\left(\mathrm{GJ} \mathrm{ha}^{-1}\right)\end{array}$} & \multicolumn{2}{|c|}{$\begin{array}{l}\text { Output } \\
\left(\mathrm{GJ} \mathrm{ha}^{-1}\right)\end{array}$} & \multicolumn{2}{|c|}{$\begin{array}{c}\text { Producción de energía } \\
\text { neta }\left(\mathrm{GJ} \mathrm{ha}^{-1}\right)\end{array}$} & \multicolumn{2}{|c|}{$\begin{array}{l}\text { Eficiencia } \\
\text { energética }\end{array}$} \\
\hline & $\mathrm{T} 2$ & T3 & $\mathrm{T} 2$ & T3 & $\mathrm{T} 2$ & T3 & $\mathrm{T} 2$ & T3 \\
\hline 2000 & 16,2 & 16,2 & - & - & $-16,2$ & $-16,2$ & - & - \\
\hline 2002 & 9,3 & 9,3 & 482,9 & 482,9 & 473,6 & 473,6 & 51,9 & 51,9 \\
\hline 2004 & 9,3 & - & 272,6 & - & 263,3 & - & 29,3 & - \\
\hline 2005 & - & 10,6 & - & 413,7 & - & 403,1 & - & 39,0 \\
\hline 2006 & 9,3 & - & 194,7 & - & 185,4 & - & 20,9 & - \\
\hline 2008 & 13,4 & 14,7 & 486,8 & 850,1 & 473,4 & 835,4 & 36,3 & 57,8 \\
\hline Total & 57,5 & 50,8 & $1.437,0$ & $1.746,7$ & $1.379,5$ & $1.695,9$ & - & - \\
\hline Media & 7,2 & 6,3 & 179,6 & 218,3 & 172,4 & 212,0 & 25,0 & 34,7 \\
\hline
\end{tabular}

\section{DISCUSIÓN}

Producción de biomasa. El estudio de las características productivas de la especie Populus deltoides clon Lux en el litoral de la Toscana (ambiente mediterráneo), caracterizado por la presencia de suelos bien dotados de elementos nutritivos y materia orgánica, y en los cuales el factor agua generalmente no representa un factor limitante debido a la presencia de una capa freática alta, ha puesto en evidencia niveles aceptables de producción de materia seca, con valores medios de 9,2 y11,5 $\mathrm{Mg}_{\text {ha-1 }} \mathrm{y}^{\text {año-1 }}{ }^{-1}$ para el turno bienal y el trienal, respectivamente. El turno bienal presenta una disminución progresiva de la producción del 2002 al 2006, mientras que en el último bienio, del 2006 al 2008, la productividad aumentó un 150 \%. La misma dinámica se observó en el turno trienal donde del 2005 al 2008, correspondiendo con el último corte, la cantidad de biomasa aumentó casi un $100 \%$ pasando de 22 a $45 \mathrm{Mg} \mathrm{ha}^{-1}$. La escasa productividad registrada en los años del 2004 al 2006, podría ser debida al prolongado periodo de sequía que caracterizó aquellos años en el área de estudio. De hecho, en los meses de mayor desarrollo del cultivo (de mayo a agosto), se registró una media de $120 \mathrm{~mm}$, valor considerado bajo para el cultivo de especies de Populus (Bonari y Villani 2004), y que pudo afectar negativamente al desarrollo del cultivo. Al contrario, la estación de crecimiento vegetativo precedente a la última cosecha efectuada en el 2008 , se caracterizó por abundantes precipitaciones (236 $\mathrm{mm}$ de mayo a agosto) lo cual podría haber favorecido el desarrollo del cultivo.

Comparando los resultados obtenidos con los encontrados en la bibliografía, se puede observar que la cosecha obtenida en este estudio es inferior. De hecho, para turnos bienales Rafaschieri (1999) obtuvo producciones variables entre 16 y $20 \mathrm{Mg} \mathrm{ha}^{-1}$ año $^{-1}$ mientras que para turnos de corta variables entre 3 y 5 años Kauter et al. (2003) utilizando Populus sp. obtuvieron producciones entre $10 \mathrm{y}$
$12 \mathrm{Mg} \mathrm{ha}^{-1} \mathrm{año}^{-1}$. Las diferencias encontradas podrían ser debidas a que en este estudio se ha utilizado el clon Lux que no ha sido seleccionado para la producción de biomasa con fines energéticos, sino que es un clon utilizado para la producción de papel. Además, estos resultados más bajos podrían deberse al tipo de terreno sobre el cual ha sido realizado el ensayo, el cual es un terreno arenoso (más del 45 $\%$ de arena) y por lo tanto poco adaptado a retener el agua meteorológica obligando al cultivo a soportar periodos de estrés hídrico durante la época estival, cuando la capa freática alcanza los niveles más bajos.

Balance energético. Los resultados obtenidos, comparables con los extraídos en otros estudios (Dubuisson y Sintzoff 1998, Matthews 2001), muestran que el cultivo de $P$. deltoides clon Lux tiene un balance energético positivo, y que la energía neta producida y la eficiencia energética varían en función del turno de corta. Además, los resultados de este estudio demuestran cómo la adopción de turnos de corta más largos mejora la eficiencia energética del sistema de cultivo y, por otro lado, disminuyen los inputs energéticos, entre los que destacan el uso de fertilizantes, lo que produce un menor impacto ambiental ligado a la eventual dispersión de nitratos y fosfatos en el sistema a causa de fenómenos de lixiviación.

La eficiencia energética registrada en el cultivo de P. deltoides clon Lux con fines energéticos tiene un valor máximo de 34,7 para el turno trienal. Los valores relativos a la eficiencia energética obtenidos en este estudio confirman los resultados obtenidos por varios autores, en cuanto a una alta variabilidad en este parámetro (de 20 a 55) en función de la gestión del cultivo y en particular en relación a los límites asumidos para la realización del balance (Matthews 2001, Heller 2003, Nassi o Di Nasso et al. 2010). Otros autores obtienen valores medios como los obtenidos en este estudio (Pontailler et al. 1999) y otros valores más elevados (Laureysens 2004). Esta variación puede ser debida a las diferentes 
condiciones edafo-climáticas y los diferentes clones usados, ambos factores muy importantes en el establecimiento de cultivos de corta rotación con fines energéticos (Ceulemans y Deraedt 1999). En este estudio, el hecho de usar un clon seleccionado para la producción de papel y no de biomasa influyó bajando la eficiencia energética obtenida.

Observando la cantidad de energía requerida para los dos turnos de rotación que se comparan en el estudio, destaca el hecho que los costos energéticos después del primer año de plantación vienen representados por la cosecha, la fertilización post-cosecha y la recuperación del terreno al finalizar el ciclo. De este modo la diferencia entre ambos sistemas es simplemente el número de cosechas en el periodo del 2000 al 2008. De hecho, el turno bienal se cosechó cuatro veces mientras que el trianual sólo tres veces; además el gasto energético en la cosecha en plantas de dos $\mathrm{y}$ tres años es diferente, por lo que este hecho también incidió en el costo energético del sistema.

De todas formas, es difícil comparar valores obtenidos de diferentes experimentos ya que las metodologías adoptadas difieren de un estudio a otro. Matthews (2001), para un cultivo de Populus sp. con destino energético y con turno de corta trienales en un ciclo de 16 años, obtuvo valores de energía neta y eficiencia energética más bajos que los obtenidos en este estudio (188 GJ ha- ${ }^{-1}$ año $^{-1}$ y 29 , respectivamente), en función de la cosecha que ha sido menor. Estas comparaciones confirman el hecho de que el manejo del cultivo (particularmente la fertilización) tiene una gran influencia en el balance energético (Mead 2006). Aun así, el balance energético a orilla de camino en el cultivo de $P$. deltoides clon Lux con destinación energética, en un ciclo de ocho años, con turnos de corta bienal y trienal ha resultado positivo, así como la eficiencia energética, siendo más eficiente el turno trienal respecto al bienal.

\section{REFERENCIAS}

Acaraglu MS. 2005. The cultivation and energy balance of Miscanthus Formula Not Shown giganteus production in Turkey. Biomass and Bioenergy 29: 42-48.

Angelini LG, L Ceccarini, E Bonari. 2005. Biomass yield and energy balance of giant reed (Arundo donax L.) cropped in central Italy as related to different management practices. European Journal of Agronomy 22: 375-389.

Baettig R, M Yáñez, M Albornoz. 2010. Cultivos dendroenergéticos de híbridos de álamo para la obtención de biocombustibles en Chile: Estado del arte. Bosque 31(2): 89-99.

Boehmel C, I Lewandowski, W Claupein. 2008. Comparing annual and perennial energy cropping systems with different management intensities. Agricultural systems 96: 224236.

Bonari E, A Peruzzi, M Mazzoncini, N Silvestri. 1992. Valutazione energetiche di sistemi produttivi a diverso livello di intensificazione colturale. L'Informatore Agrario 1.

Bonari E, N Silvestri, S Pampana. 1999. Sisco: a practical tool to assess alternative cropping systems' performances. Proceedings International Symposium Modelling Cropping Systems. Lleida (Spain): 285-286.
Bonari E, R Villani. 2004. La produzione di biomassa a destinazione energetica da S.R.F. di pioppo. Prime esperienze in Toscana. Convegno di Studio: Le biomasse agricole e forestali nello scenario energetico nazionale. Progetto Fuoco. Verona 18-19 Marzo 2004.

Ceulemans R, W Deraedt. 1999. Production physiology and growth potential of poplars under short-rotation forestry culture. Forest Ecology and Management 121: 9-23.

Dillen SY, SN Djomo, N Al Afas, S Vanbeveren, R Ceulemans. 2013. Biomass yield and energy balance of a short-rotation poplar coppice with multiple clones on degraded land during 16 years 5. Biomass and Bioenergy 56: 157-165.

Dubuisson X, I Sintzoff. 1998. Energy and $\mathrm{CO}_{2}$ balances in different power generation routes using wood fuel from short rotation coppice. Biomass and Bioenergy 15: 379-390.

Gasol CM, X Gabarrel, A Anton, M Rigola, J Carrasco, P Ciria, J Rieradevall. 2009. LCA of poplar bionergy system compared with Brassica carinata energy crop and natural gas in regional scenario. Biomass and Bioenergy 33: 119-129.

Heller MC, GA Keoleian, TA Volk. 2003. Life cycle assessment of a willow bioenergy cropping system. Biomass and Bioenergy 25: 147-165.

Kalk WD, KJ Hulsbergen. 1996. Methodik zur Einbeziehung des indirekten Energieverbrauchs mit Investitionsgutern in Energiebilanzen von Landwirtschaftsbetrieben. KühnArchiv 90: 41-56.

Kaltschmitt M, GA Reinhardt, T Stelzer. 1997. Life cycle analysis of biofuels under different environmental aspects. Biomass Bioenergy 12: 121-134.

Kauter D, I Lewandowski, W Claupein. 2003. Quantity and quality of harvestable biomass from Populus short rotation coppice for solid fuel use - a review of the physiological basis and management influences. Biomass and Bioenergy 24(6): 411-427.

Laureysens I, J Bogaert, R Blust, R Ceulemans. 2004. Biomass production of 17 poplar clones in a short-rotation coppice culture on a waste disposal site and its relation to soil characteristics. Forest Ecology and Management 187: 295309.

Mantineo M, GM D’Agosta, V Copani, C Patane, SL Cosentino. 2009. Biomass yield and energy balance of three perennial crops for energy use in the semi-arid Mediterranean environment. Field Crops Research 114: 204-213

Matthews RW. 2001. Modelling of energy and carbon budgets of wood fuel coppice systems. Biomass and Bioenergy 21(1): 1-19.

Mead DJ, D Pimentel. 2006. Use of energy analysis in silvicultural decisionmaking. Biomass and Bioenergy 30: 357-362.

Nassi o Di Nasso N, W Guidi, G Ragaglini, C Tozzini, E Bonari. 2010. Biomass production and energy balance of a 12 -yearold short-rotation coppice poplar stand under different cutting cycles. GCB Bioenergy 2: 89-97.

Pontailler JY, R Ceulemans, J Guittet. 1999. Biomass yield of poplar after five 2-years coppice rotations. Forestry 72: 157-163.

Rafaschieri A, M Rapaccini, G Manfrida.1999. Life cycle assessment of electricity production from poplar energy crops compared with conventional fossil fuels. Energy Conversion and Management 40: 1477-1493.

Sartori L, B Basso, M Bertocco, G Oliviero. 2005. Energy use and economic evaluation of a three year crop rotation for 
conservation and organic farming in NE Italy. Biosystems Engineering 91: 245-256.

Scholz V, W Berg, P Kaulfuss. 1998. Energy balance of Solid Biofuels. Journal of Agriculture Engineering Research 71: 263-272.

Scholz V, R Ellerbrock. 2002. The growth productivity, and environmental impact of the cultivation of energy crops on sandy soil in Germany. Biomass and Bioenergy 23: 81-92.

Sevigne E, CM Gasol, F Brun, L Rovira, JM Pagés, F Camps, J Rieredevall, X Gabarrell. 2011. Water and energy con- sumption of Pupulus spp. bioenergy systems: A case study in Southern Europe. Renewable and Sustainable Energy Reviews 15: 1133-1140.

The Royal Society. 2008. Sustainable biofuels: prospects and challenges. Policy document '1/08. ISBN 978085403662 2. London, UK. The Royal Society. 82 p.

West TO, G Marland. 2002. A synthesis of carbon sequestration, carbon emissions, and net carbon flux in agriculture: Comparing tillage practices in the United States. Agriculture, Ecosystems and Environment 91(1-3): 217-232.

Recibido: 14.01 .13

Aceptado: 14.04.14 
